\title{
Bifurcations and Synchronization of the Fractional-Order Bloch System
}

\author{
Xiaojun Liu, ${ }^{1,2}$ Ling Hong, ${ }^{2}$ Honggang Dang, ${ }^{1}$ and Lixin Yang ${ }^{1}$ \\ ${ }^{1}$ School of Mathematics and Statistics, Tianshui Normal University, Tianshui 741001, China \\ ${ }^{2}$ State Key Laboratory for Strength and Vibration of Mechanical Structures, Xian Jiaotong University, Shaanxi, Xian 710049, China \\ Correspondence should be addressed to Xiaojun Liu; flybett3952@126.com
}

Received 13 February 2017; Accepted 6 March 2017; Published 22 March 2017

Academic Editor: Mohamed A. Hajji

Copyright (c) 2017 Xiaojun Liu et al. This is an open access article distributed under the Creative Commons Attribution License, which permits unrestricted use, distribution, and reproduction in any medium, provided the original work is properly cited.

\begin{abstract}
In this paper, bifurcations and synchronization of a fractional-order Bloch system are studied. Firstly, the bifurcations with the variation of every order and the system parameter for the system are discussed. The rich dynamics in the fractional-order Bloch system including chaos, period, limit cycles, period-doubling, and tangent bifurcations are found. Furthermore, based on the stability theory of fractional-order systems, the adaptive synchronization for the system with unknown parameters is realized by designing appropriate controllers. Numerical simulations are carried out to demonstrate the effectiveness and flexibility of the controllers.
\end{abstract}

\section{Introduction}

Nowadays, fractional calculus is a hot topic in the research field. It is well known that fractional calculus has an equally long history with classical calculus. It did not attract enough attention for the absence of geometrical interpretation and applications at the initial stage of development. As the development of technology and science continues, fractional calculus has been applied in many fields, such as control theory, dynamics, mathematics, mechanics, and physics [1$5]$.

As the research of fractional calculus moves along, many nonlinear systems with fractional orders are proposed and investigated. The chaos and bifurcations which are observed in integer-order systems are also found in fractional-order ones, such as fractional versions of Duffing system, Lorenz system, and Chen system [6-11]. It is well known that the Bloch system is very important for interpretation of the underlying physical process of nuclear magnetic resonance. Recently, the fractional-order Bloch equations with and without delay were studied [12-14]. Meanwhile, physical interest in the fractional-order Bloch equation has been growing $[15,16]$ with the goal of improving the modeling of relaxation, diffusion, and perfusion in biological tissues. In [17], for the fractional-order Bloch system, the chaotic dynamics including the chaotic attractors in different system parameters sets, bifurcations with the derivative order in commensurate-order case, were analyzed. Rich dynamics such as period-doubling and subharmonic cascade routes to chaos were found for the system in the commensurate-order case. Based on these results, we want to know the bifurcations of the fractional-order Bloch system with the variation of every order in incommensurate-order case as well as every system parameter.

Motivated by the above discussed, in this paper, the bifurcations with the derivative order in incommensurateorder case and system parameters are studied firstly. A series of period-doubling bifurcations and tangent bifurcations are obtained by numerical simulations. Meanwhile, different chaotic and periodic attractors are also observed. Furthermore, based on the stability theory of fractional-order systems, the adaptive synchronization of the fraction-order systems with uncertain parameters is realized by designing appropriate controllers. Numerical simulations are carried out to demonstrate the effectiveness and flexibility of the controllers. 
The paper is organized as follows. In Section 2, the definitions for the fractional calculus and numerical algorithms for fractional differential equations are given. The bifurcations of the fractional-order Bloch system are investigated in detail in Section 3. In Section 4, the adaptive synchronization of the system is investigated. Numerical simulations are used to demonstrate the effectiveness of the controllers. Finally, we summarize the results in Section 5.

\section{Fractional Derivatives}

There are many definitions for the general fractional derivative. The three most frequently used ones are the GrunwaldLetnikov definition and the Riemann-Liouville and the Caputo definitions. It is well known that the initial conditions for the fractional differential equations with Caputo derivatives take on the same form as those for the integer-order ones, which is very suitable for practical problems. Therefore, we will use the Caputo definition for the fractional derivatives in this paper.

The Caputo fractional derivative is defined as follows:

$$
\begin{array}{r}
{ }_{a} D_{t}^{q} f(t)=\frac{1}{\Gamma(n-q)} \int_{a}^{t}(t-\tau)^{n-q-1} f^{(n)}(\tau) d \tau, \\
n-1<q<n .
\end{array}
$$

As the initial conditions for the fractional differential equations with Caputo derivatives take on the same form as those for the integer-order ones, we will use the Caputo definition for the fractional derivatives in this paper.

In the following, we will give the definitions of commensurate-order and incommensurate-order fractionalorder systems [18].

Definition 1. For a fractional-order system, which can be described by $D^{\mathbf{q}} \mathbf{x}=f(\mathbf{x})$, where $\mathbf{x}=\left(x_{1}, x_{2}, \ldots, x_{n}\right)^{\mathrm{T}}$ is the state vector, $\mathbf{q}=\left(q_{1}, q_{2}, \ldots, q_{n}\right)^{\mathrm{T}}$ is the fractional derivative orders vector, and $q_{i}>0$. The fractional-order system is commensurate-order when all the derivative orders satisfy $q_{1}=q_{2}=\cdots=q_{n}$; otherwise it is an incommensurate-order system.

Compared with the numerical algorithm for solving an ordinary differential equation, the numerical solution of a fractional differential equation is not easy to obtain. There are two approximation methods which can frequently be used for numerical computation on chaos and bifurcations with fractional differential equations. One is an improved version of Adams-Bashforth-Moulton algorithm based on the predictor-correctors scheme [19-21], which is a time domain approach. The other is a method, known as frequency domain approximation [22], based on numerical analysis of fractional-order systems in the frequency domain.

Simulation of fractional-order systems using the time domain methods is complicated and, due to long memory characteristics of these systems, requires a very long simulation time but on the other hand, it is more accurate [23]. Therefore, we employ the improved predictor-corrector algorithm for fractional-order differential equations in this paper.

\section{A Fractional-Order Bloch System}

The Bloch system is usually used to describe an ensemble of spins. The integer-order and fractional-order Bloch systems were studied in [17]. In this section, the bifurcations of the fractional-order Bloch system with the variation of different system parameters and derivative orders will be investigated.

The fractional-order Bloch system can be described as follows:

$$
\begin{aligned}
& D^{q_{1}} x=\delta y+\gamma z(x \sin (c)-y \cos (c))-\frac{x}{\Gamma_{2}} \\
& D^{q_{2}} y=-\delta x-z+\gamma z(x \cos (c)+y \sin (c))-\frac{y}{\Gamma_{2}} \\
& D^{q_{3}} z=y-\gamma \sin (c)\left(x^{2}+y^{2}\right)-\frac{z-1}{\Gamma_{1}}
\end{aligned}
$$

where $x, y, z$ are the state variables, $q_{1}, q_{2}, q_{3}$ the derivative orders, and $\delta, \gamma, c, \Gamma_{1}, \Gamma_{2}$ the system parameters. When the orders $q_{1}=q_{2}=q_{3}=q=0.99$, system (2) has a chaotic attractor with system parameters $\delta=1.26, \gamma=10, c=$ $0.7764, \Gamma_{1}=0.5, \Gamma_{2}=0.25$, which is plotted in Figure 1 . The initial conditions for the numerical simulation are $x(0)=0.1$, $y(0)=0.1, z(0)=0.1$ and also used in the rest of the paper.

3.1. Bifurcations with the Variation of the Orders. When the system parameters are fixed as $\delta=1.26, \gamma=10, c=$ $0.7764, \Gamma_{1}=0.5, \Gamma_{2}=0.25$, and the orders $q_{2}=1$, $q_{3}=1$, the bifurcation of system (2) as the order $q_{1}$ is varied is depicted in Figure 2(a). From this, it is clear that the system has a long period-1 window when $q_{1}$ is slightly less than 0.72 . As the order $q_{1}$ further increases, an evolution procedure of system (2) through period-doubling route to chaos is obtained. Meanwhile, to verify the chaotic behaviors for the system, the corresponding largest Lyapunov exponent (LLE for short) diagram by the algorithm of small data sets is shown in Figure 2(b). Phase portraits and the corresponding time series diagrams are shown in Figures 3(a)-3(h), from which we can see that the system has period-1, period-2, period-4, and chaos for different values of the order $q_{1}$. The route out of chaos for system (2) is tangent bifurcation when $q_{1}=0.833$. There are three limit cycles coexisting until the secondary period-doubling bifurcations occur.

When the system parameters are fixed as the same values, and the orders $q_{1}=1, q_{3}=1$, the affection of the variation of order $q_{2} \in[0.5,1]$ for the system is showed in Figure 4(a). It is clear that a series of period-doubling and tangent bifurcations can also be seen from the figure. Meanwhile, the corresponding LLE diagram is shown in Figure 4(b). As the order $q_{3}$ is varied, the bifurcations of the system are similar to that of $q_{2}$, so more details are not displayed in here.

3.2. Bifurcations with the Variation of the System Parameters. When the other parameters are taken as $\gamma=10, c=0.7764$, 


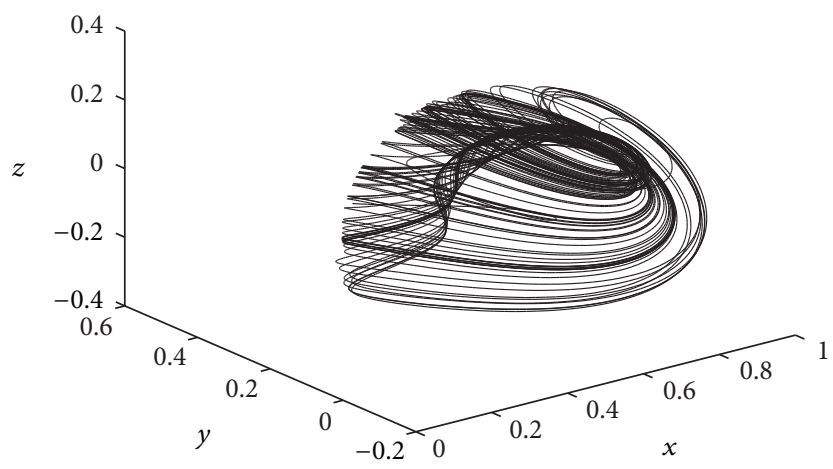

FIGURE 1: The chaotic attractor of system (2).

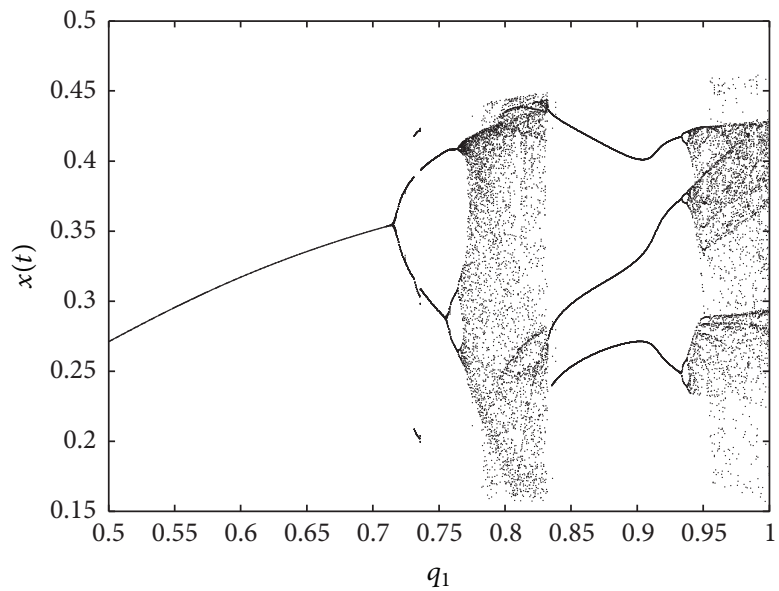

(a) The bifurcation diagram

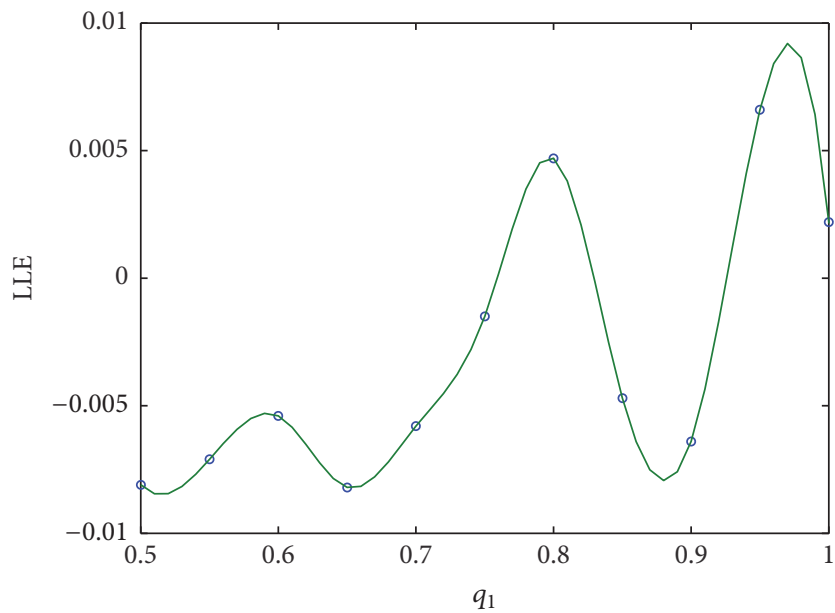

(b) The corresponding LLE

FIGURE 2: The bifurcation and the corresponding LLE diagrams of system (2) with the order $q_{1} \in[0.5,1]$.

$\Gamma_{1}=0.5, \Gamma_{2}=0.25$, and the orders $q_{1}=q_{2}=q_{3}=$ 0.98 , the bifurcation and the corresponding LLE diagrams of system (2) varying the parameter $\delta$ are depicted in Figure 5. With the increase of the parameter $\delta$ from -1.26 , the system is period-1 firstly when $\delta \in[-1.26,-0.38]$. Then system (2) is period-2 motion until a series of period-doubling bifurcations occur. The phase portraits and the corresponding time series diagrams of system (2) with different values of the parameter $\delta$ are showed in Figure 6, from which it is clear that the system has only one limit cycle for $\delta=-1.26$, two cycles for $\delta=-0.7$, four cycles for $\delta=0.7$, and six cycles for $\delta=0.8$.

When $\delta=1.26$, the bifurcation and the corresponding LLE diagrams of the system with the variation of $\gamma$ are depicted in Figure 7. From this, we can see that a series of period-doubling and tangent bifurcations can be observed. Meanwhile, the bifurcation and the corresponding LLE diagrams of system (2) with the system parameters $c, \Gamma_{1}$, and $\Gamma_{2}$ are also obtained by numerical simulations, respectively (see Figure 8). It is clear that the bifurcations of the system with $c$ and $\Gamma_{2}$ are similar to that of the parameter $\gamma$. The system undergoes a series of period-doubling bifurcations when the parameter $\Gamma_{1}$ is varied and the alternation between the period and chaos.

\section{Adaptive Synchronization}

In this section, the adaptive synchronization for system (2) with uncertain parameters will be investigated.

For simplicity, system (2) in commensurate-order case is taken as the drive system and can be rewritten as follows:

$$
\begin{aligned}
& D^{q} x_{1}=\delta y_{1}+\gamma z_{1}\left(x_{1} \sin (c)-y_{1} \cos (c)\right)-\frac{x_{1}}{\Gamma_{2}} \\
& D^{q} y_{1}=-\delta x_{1}-z_{1}+\gamma z_{1}\left(x_{1} \cos (c)+y_{1} \sin (c)\right)-\frac{y_{1}}{\Gamma_{2}} \\
& D^{q} z_{1}=y_{1}-\gamma \sin (c)\left(x_{1}^{2}+y_{1}^{2}\right)-\frac{z_{1}-1}{\Gamma_{1}},
\end{aligned}
$$

where $\delta$ and $\gamma$ are uncertain parameters which are needed to be identified. And the response system is described by the following differential equations:

$$
\begin{aligned}
D^{q} x_{2}= & \widetilde{\delta} y_{2}+\widetilde{\gamma} z_{2}\left(x_{2} \sin (c)-y_{2} \cos (c)\right)-\frac{x_{2}}{\Gamma_{2}}+u_{1} \\
D^{q} y_{2}= & -\widetilde{\delta} x_{2}-z_{2}+\widetilde{\gamma} z_{2}\left(x_{2} \cos (c)+y_{2} \sin (c)\right)-\frac{y_{2}}{\Gamma_{2}} \\
& +u_{2}
\end{aligned}
$$




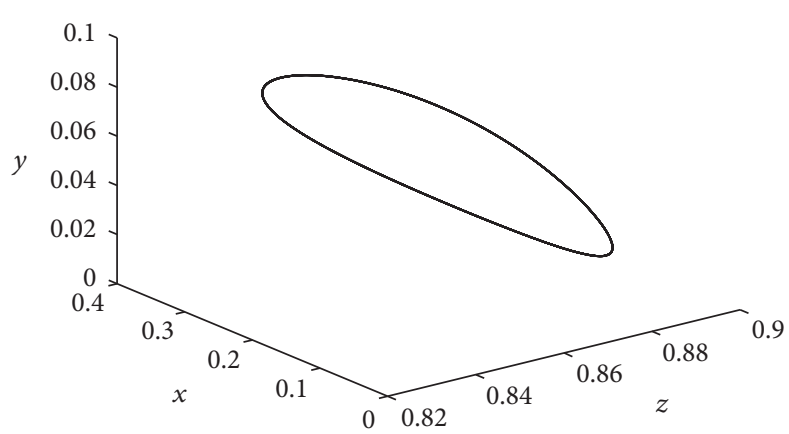

(a) The phase portrait when $q_{1}=0.5$

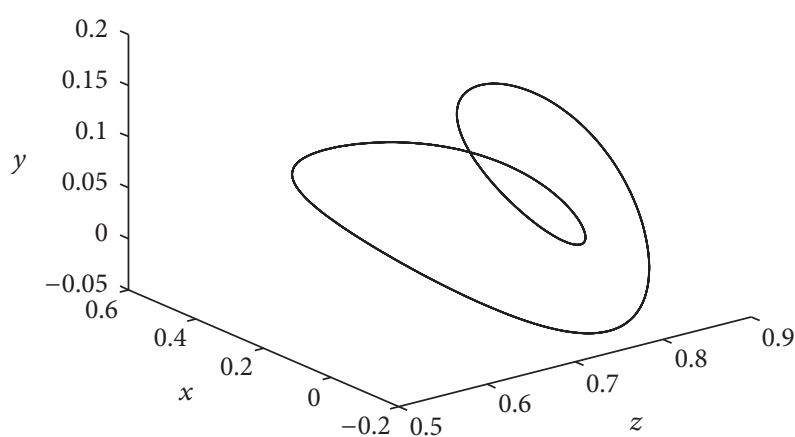

(c) The phase portrait when $q_{1}=0.75$

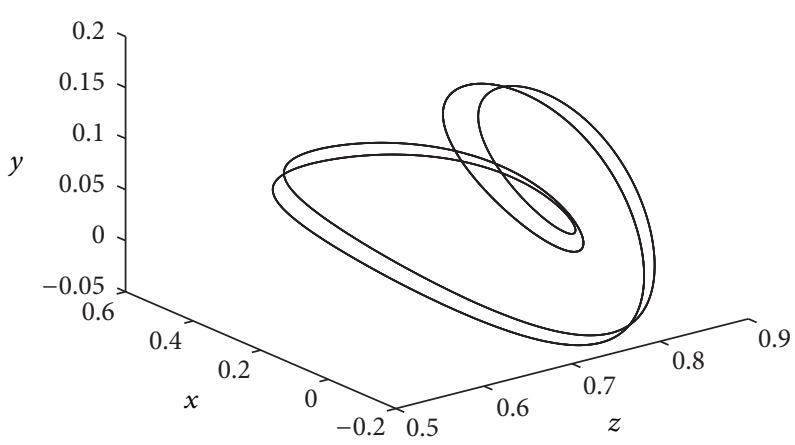

(e) The phase portrait when $q_{1}=0.76$

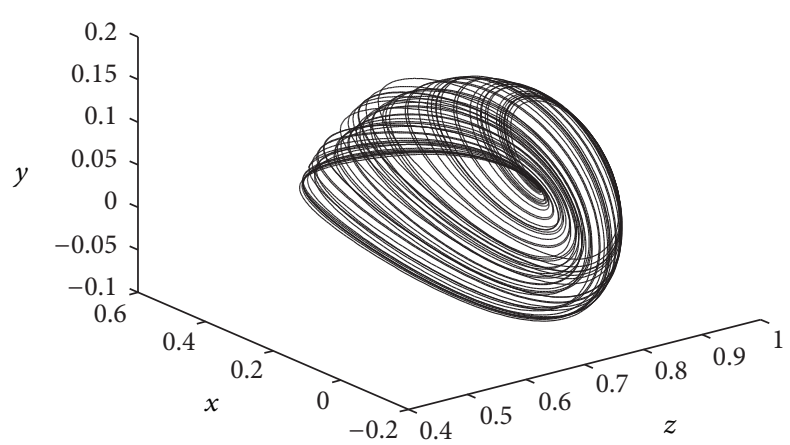

(g) The phase portrait when $q_{1}=0.78$

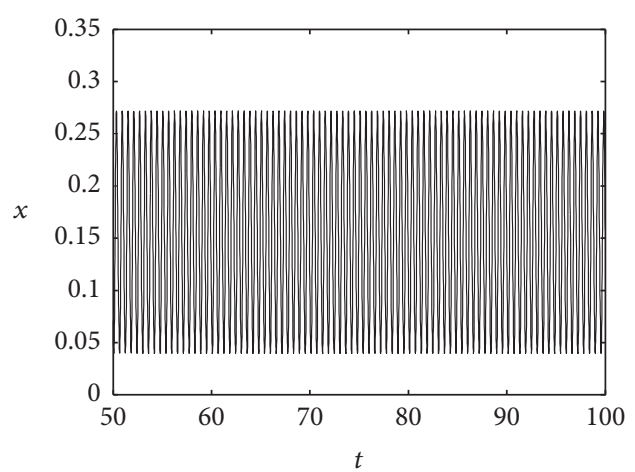

(b) The time series diagram when $q_{1}=0.5$

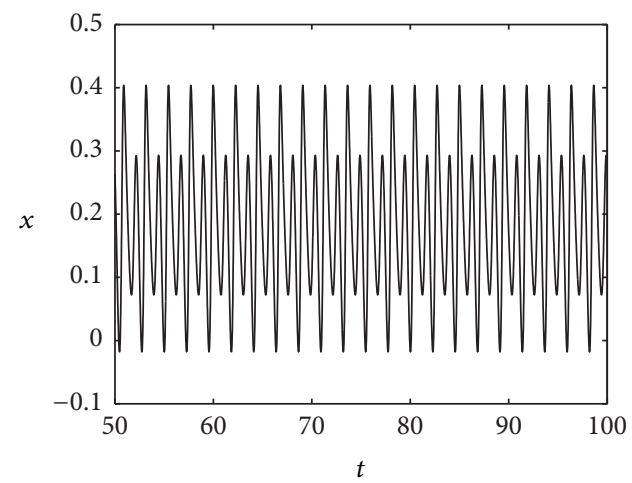

(d) The time series diagram when $q_{1}=0.75$

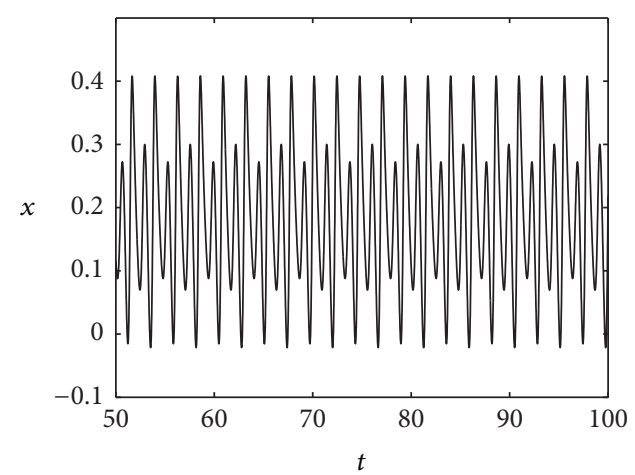

(f) The time series diagram when $q_{1}=0.76$

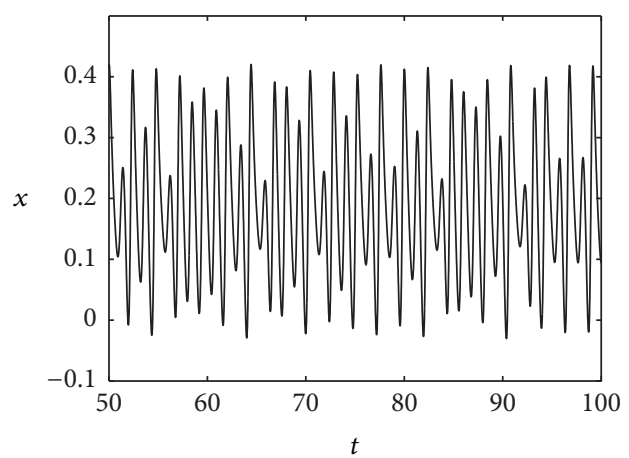

(h) The time series diagram when $q_{1}=0.78$

FIgURE 3: The phase portraits and time series diagrams of system (2) with different values of the order $q_{1}$. 


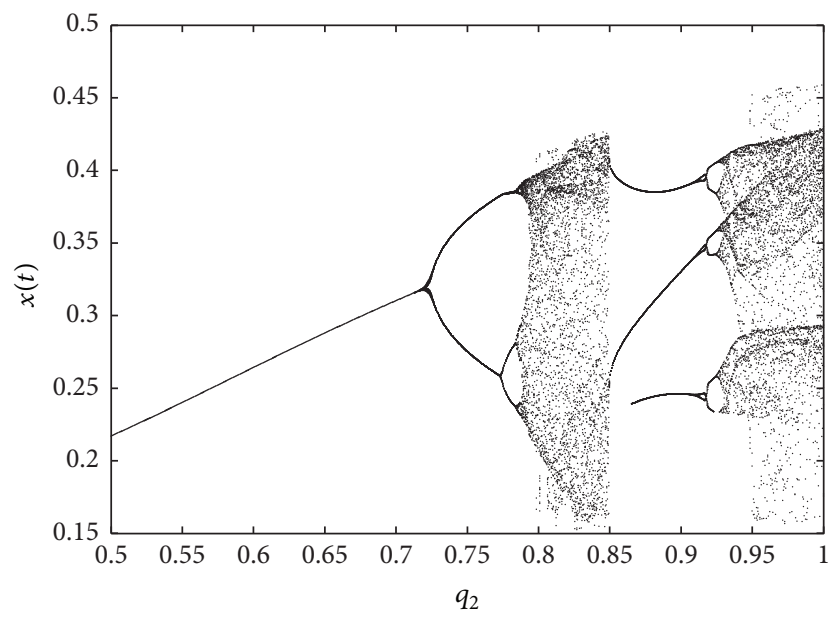

(a) The bifurcation diagram

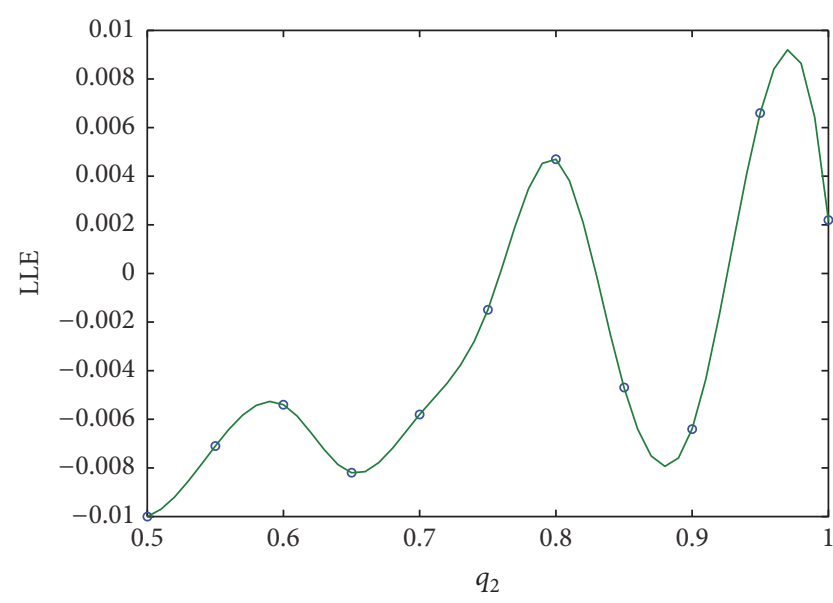

(b) The corresponding LLE

FIGURE 4: The bifurcation and the corresponding LLE diagrams of system (2) with the order $q_{2} \in[0.5,1]$.

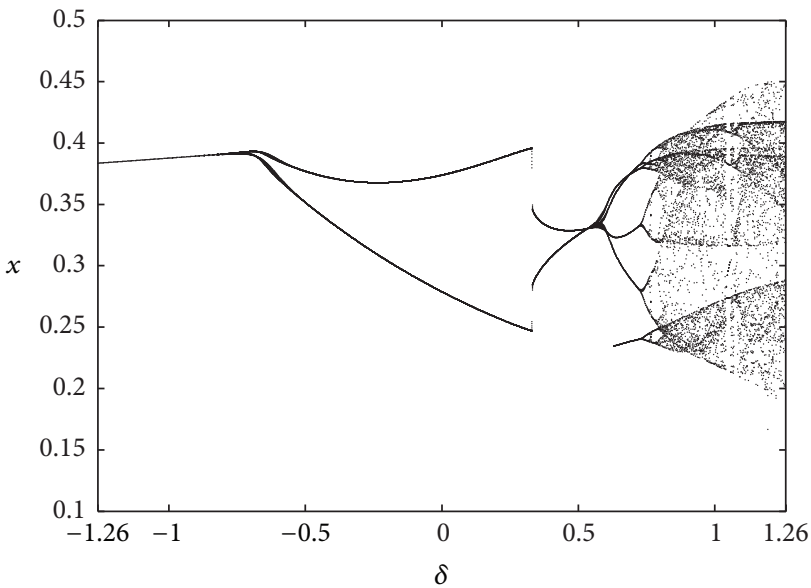

(a) The bifurcation diagram

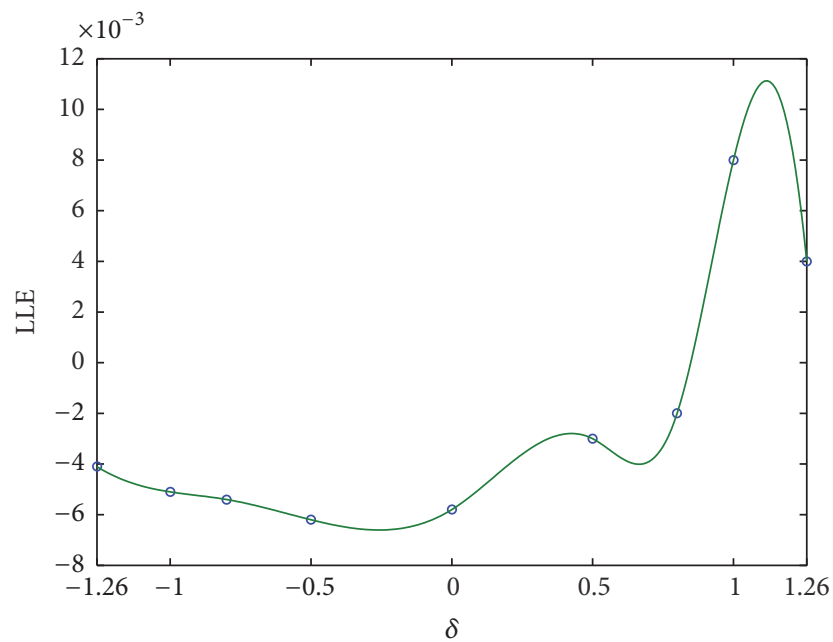

(b) The corresponding LLE

FIgURE 5: The bifurcation and the corresponding LLE diagrams of system (2) with the parameter $\delta \in[-1.26,1.26]$.

$$
D^{q} z_{2}=y_{2}-\tilde{\gamma} \sin (c)\left(x_{2}^{2}+y_{2}^{2}\right)-\frac{z_{2}-1}{\Gamma_{1}}+u_{3},
$$

where $u_{1}, u_{2}$, and $u_{3}$ are the synchronization controllers and $\widetilde{\delta}, \widetilde{\gamma}$ are the estimations of unknown parameters. Then, the synchronization error variables are defined as $e_{1}=x_{2}-$ $x_{1}, e_{2}=y_{2}-y_{1}, e_{3}=z_{2}-z_{1}$, and estimation errors of uncertain parameters $e_{\delta}=\widetilde{\delta}-\delta, e_{\gamma}=\widetilde{\gamma}-\gamma$. By subtracting system (3) from (4), the error dynamical system is obtained, which is given as follows:

$$
\begin{aligned}
D^{q} e_{1}= & \widetilde{\delta} e_{2}+y_{1} e_{\delta}+\widetilde{\gamma} e_{3}\left(x_{2} \sin (c)-y_{2} \cos (c)\right) \\
& +\widetilde{\gamma} z_{1}\left(e_{1} \sin (c)-e_{2} \cos (c)\right) \\
& +e_{\gamma} z_{1}\left(x_{1} \sin (c)-y_{1} \cos (c)\right)-\frac{e_{1}}{\Gamma_{2}}+u_{1}
\end{aligned}
$$

$$
\begin{aligned}
D^{q} e_{2}= & -\tilde{\delta} e_{1}-x_{1} e_{\delta}-e_{3}+\widetilde{\gamma} e_{3}\left(x_{2} \cos (c)+y_{2} \sin (c)\right) \\
& +\widetilde{\gamma} z_{1}\left(e_{1} \cos (c)+e_{2} \sin (c)\right) \\
& +e_{\gamma} z_{1}\left(x_{1} \cos (c)+y_{1} \sin (c)\right)-\frac{e_{2}}{\Gamma_{2}}+u_{2} \\
D^{q} e_{3}= & e_{2}-\tilde{\gamma}\left(e_{1}\left(x_{1}+x_{2}\right)+e_{2}\left(y_{1}+y_{2}\right)\right) \sin (c) \\
& -e_{\gamma}\left(x_{1}^{2}+y_{1}^{2}\right) \sin (c)-\frac{e_{3}}{\Gamma_{1}}+u_{3} .
\end{aligned}
$$

In order to realize the synchronization of the drive and response systems, the controllers should be designed properly. Therefore, the following criterion is presented to ensure system (3) effectively synchronizes system (4). 


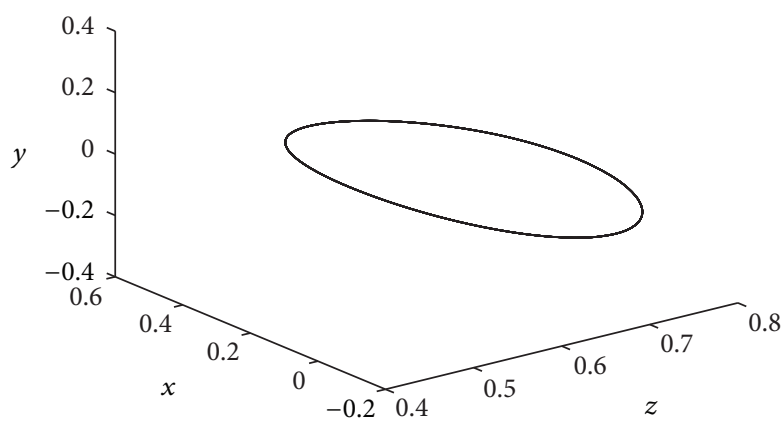

(a) The phase portrait when $\delta=-1.26$

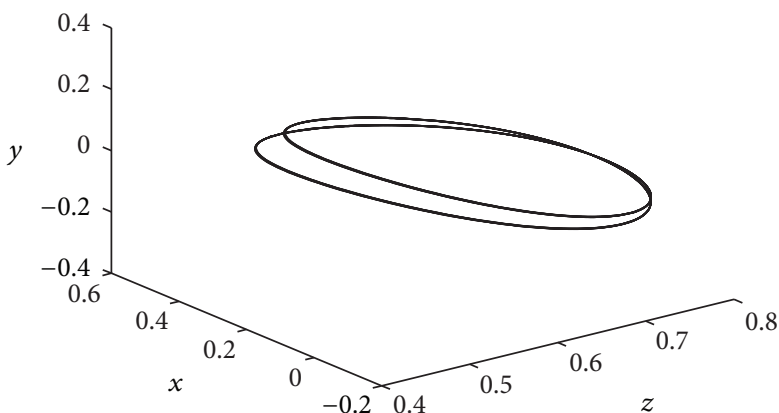

(c) The phase portrait when $\delta=-0.7$

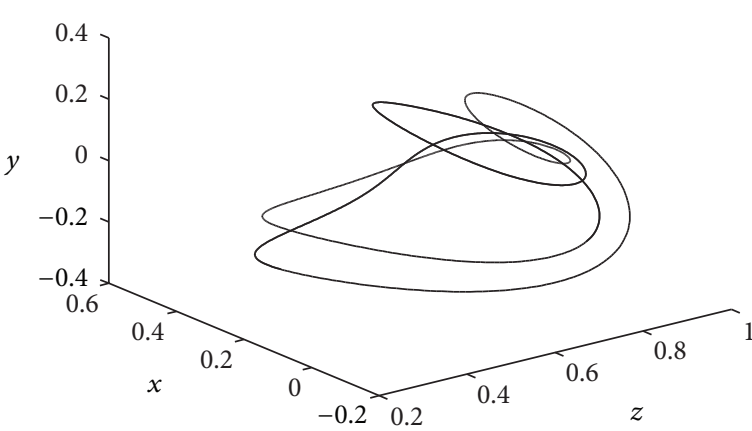

(e) The phase portrait when $\delta=0.7$

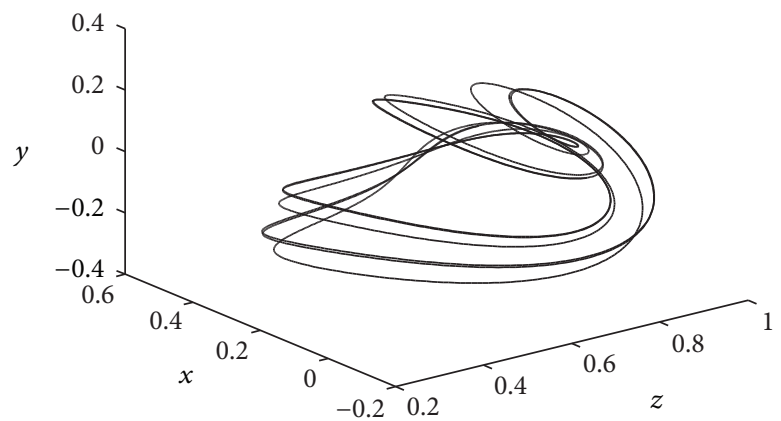

(g) The phase portrait when $\delta=0.8$

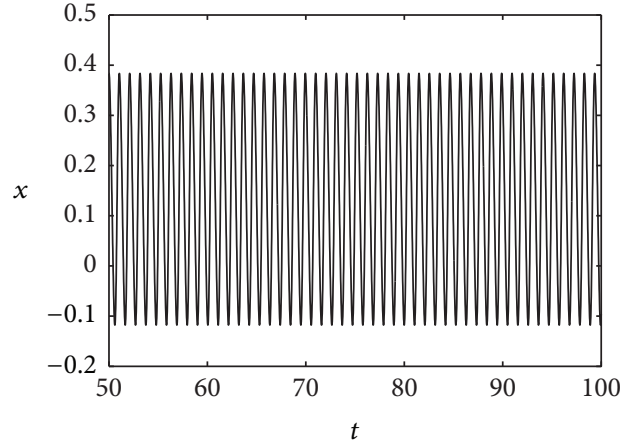

(b) The time series diagram when $\delta=-1.26$

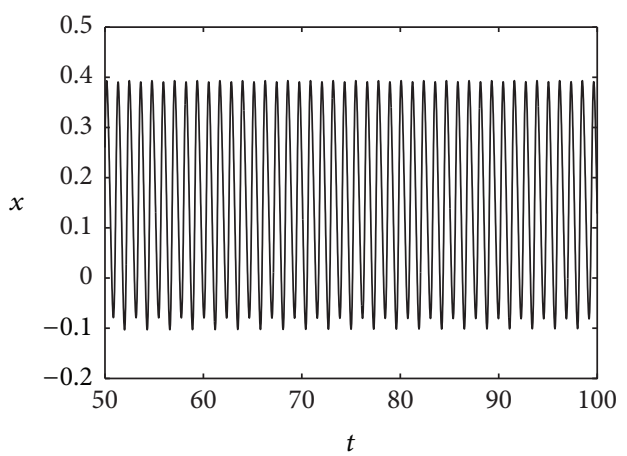

(d) The time series diagram when $\delta=-0.7$

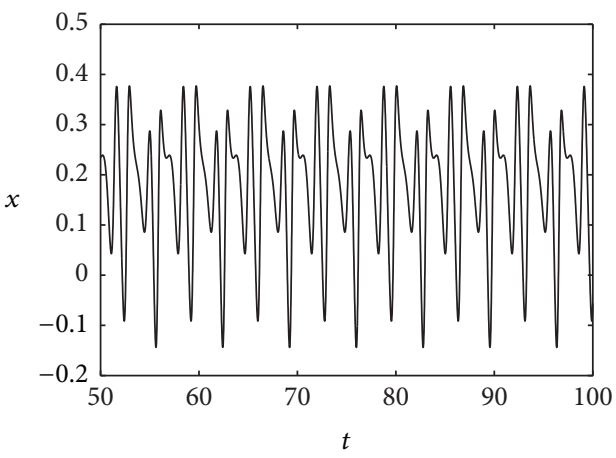

(f) The time series diagram when $\delta=0.7$

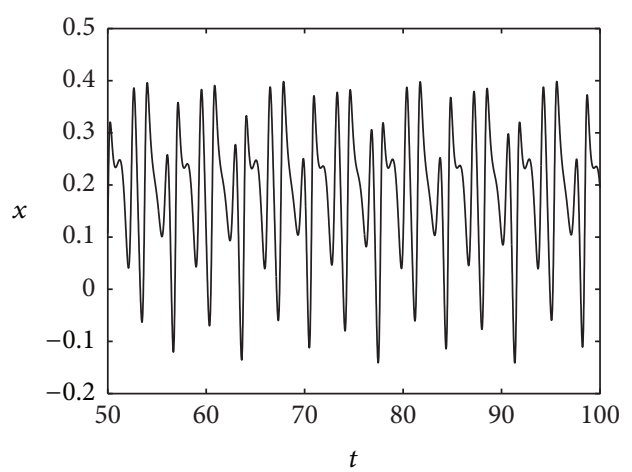

(h) The time series diagram when $\delta=0.8$

FIGURE 6: The phase portraits and time series diagrams of system (2) with different values of the parameter $\delta$. 


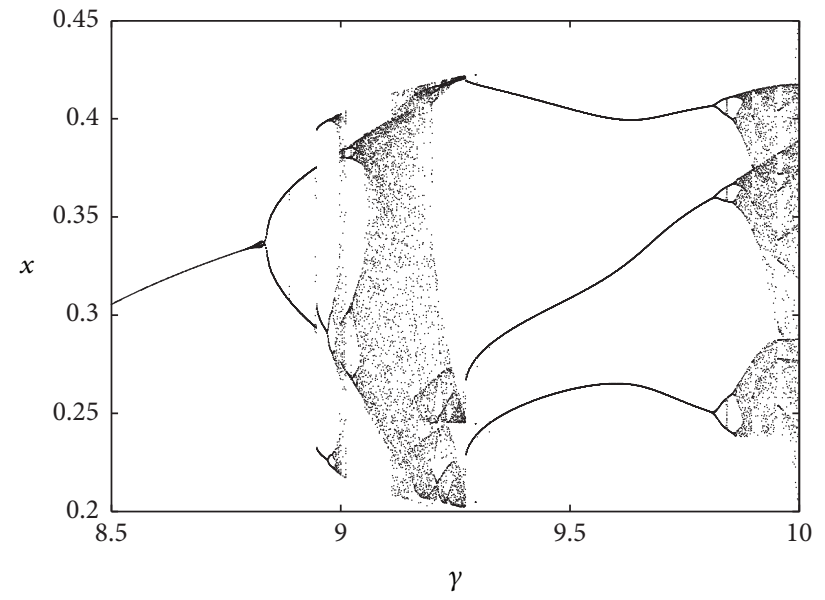

(a) The bifurcation diagram

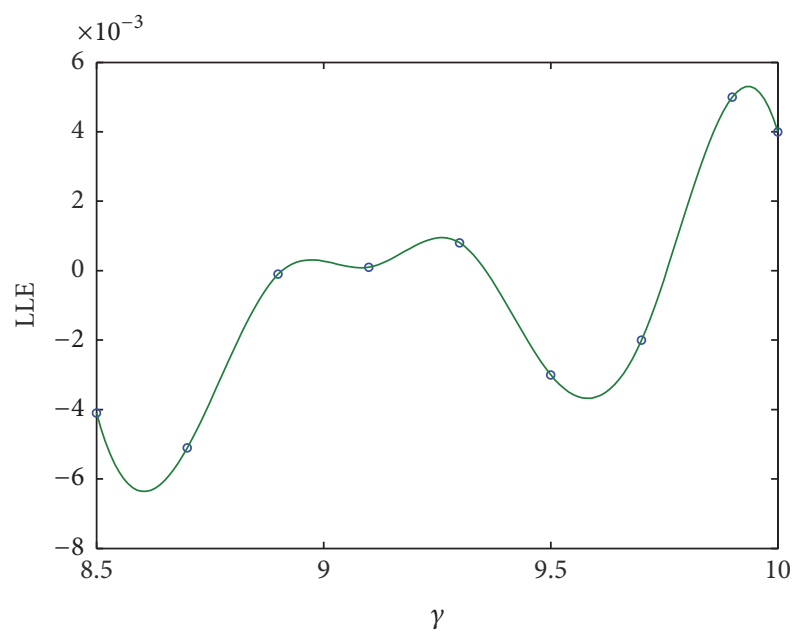

(b) The corresponding LLE

FIGURE 7: The bifurcation and the corresponding LLE diagrams of system (2) with the parameter $\gamma \in[8.5,10]$.

Theorem 2. Adaptive synchronization between systems (3) and (4) is realized when the controllers and laws of the uncertain parameter are designed as follows:

$$
\begin{aligned}
u_{1}= & -\widetilde{\delta} e_{2}-\tilde{\gamma} e_{3}\left(x_{2} \sin (c)-y_{2} \cos (c)\right) \\
& -\widetilde{\gamma} z_{1}\left(e_{1} \sin (c)-e_{2} \cos (c)\right)+\left(\frac{1}{\Gamma_{2}}-1\right) e_{1} \\
u_{2}= & \widetilde{\delta} e_{1}+e_{3}-\tilde{\gamma} e_{3}\left(x_{2} \cos (c)+y_{2} \sin (c)\right) \\
& -\tilde{\gamma} z_{1}\left(e_{1} \cos (c)+e_{2} \sin (c)\right)+\left(\frac{1}{\Gamma_{2}}-1\right) e_{2} \\
u_{3}= & -e_{2}+\widetilde{\gamma}\left(e_{1}\left(x_{1}+x_{2}\right)+e_{2}\left(y_{1}+y_{2}\right)\right) \sin (c) \\
& +\left(\frac{1}{\Gamma_{1}}-1\right) e_{3}, \\
D^{q} \widetilde{\delta}= & -y_{1} e_{1}+x_{1} e_{2} \\
D^{q} \tilde{\gamma}= & -z_{1}\left(x_{1} \sin (c)-y_{1} \cos (c)\right) e_{1} \\
& -z_{1}\left(x_{1} \cos (c)+y_{1} \sin (c)\right) e_{2} \\
& +\left(x_{1}^{2}+y_{1}^{2}\right) \sin (c) e_{3} .
\end{aligned}
$$

where
Proof. Firstly, controllers (6) are substituted into system (5), and then the error dynamical system is expressed as

$$
\begin{aligned}
& D^{q_{1}} e_{1}=y_{1} e_{\delta}+e_{\gamma} z_{1}\left(x_{1} \sin (c)-y_{1} \cos (c)\right)-e_{1} \\
& D^{q_{1}} e_{2}=-x_{1} e_{\delta}+e_{\gamma} z_{1}\left(x_{1} \cos (c)+y_{1} \sin (c)\right)-e_{2} \\
& D^{q_{1}} e_{3}=-e_{\gamma}\left(x_{1}^{2}+y_{1}^{2}\right) \sin (c)-e_{3} .
\end{aligned}
$$

By combining systems (8) and (7), we can get

$$
\begin{aligned}
& \left(D^{q} e_{1}, D^{q} e_{2}, D^{q} e_{3}, D^{q} e_{\delta}, D^{q} e_{\gamma}\right)^{\mathrm{T}} \\
& =A\left(e_{1}, e_{2}, e_{3}, e_{\delta}, e_{\gamma}\right)^{\mathrm{T}},
\end{aligned}
$$

A

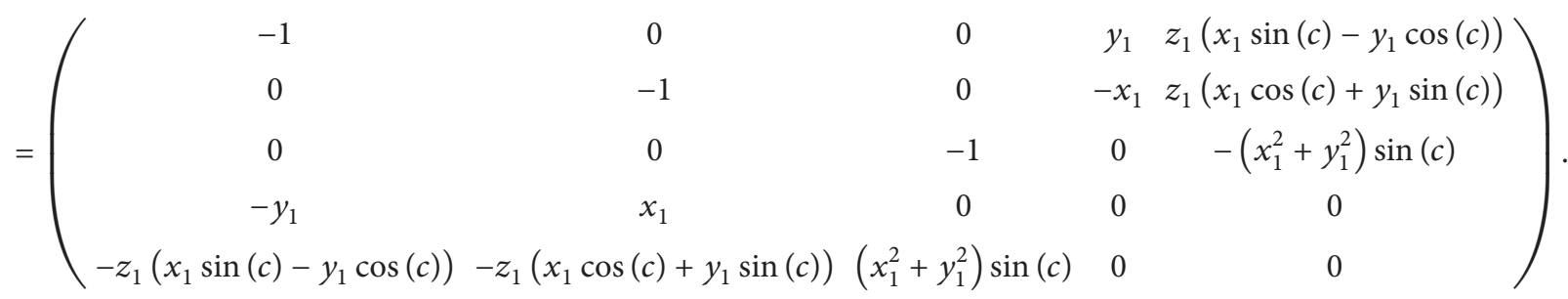




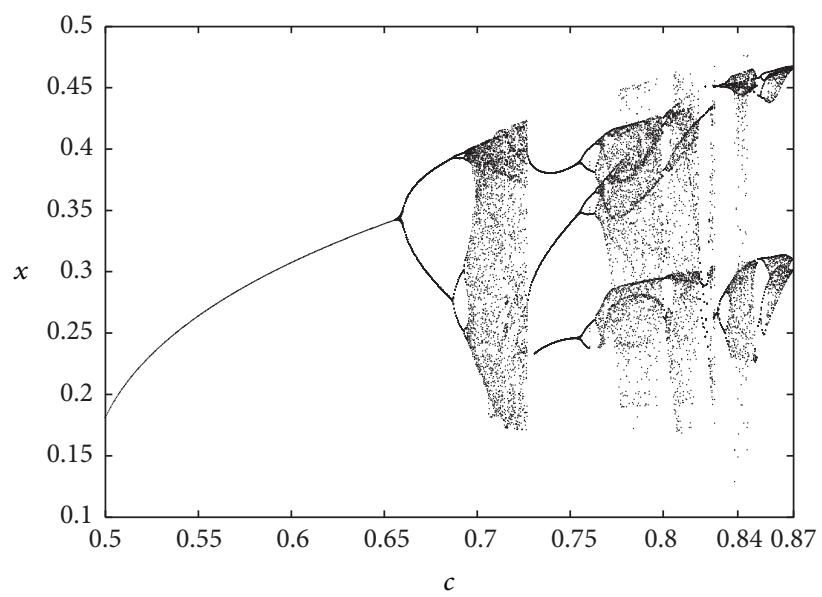

(a) The bifurcation diagram for $c \in[0.5,0.87]$

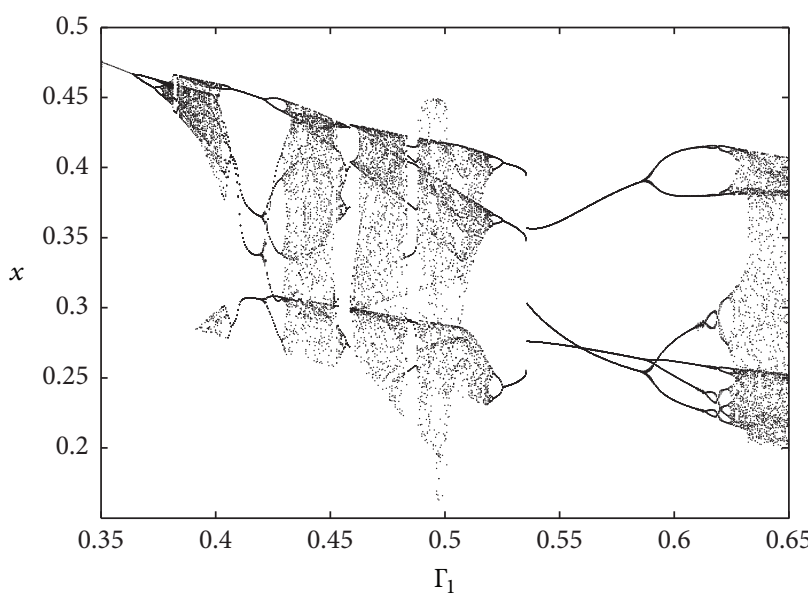

(c) The bifurcation diagram for $\Gamma_{1} \in[0.35,0.65]$

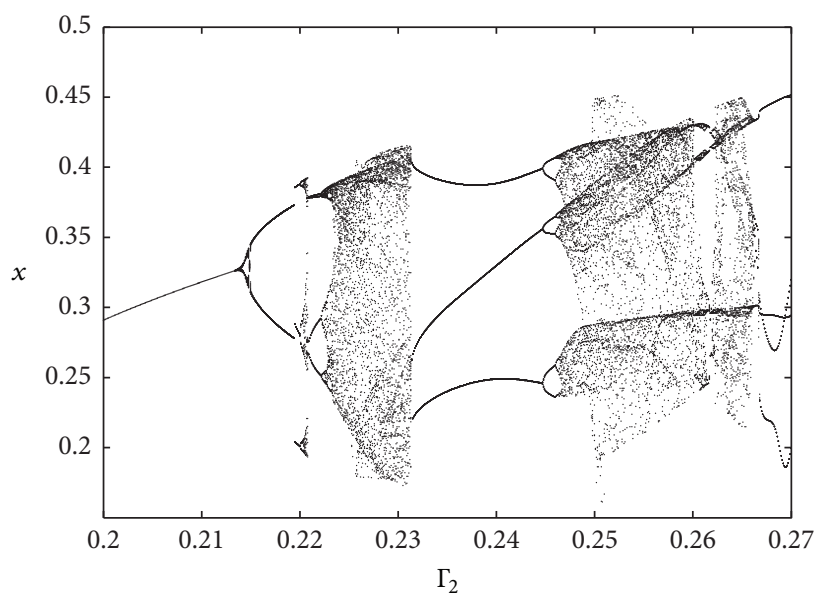

(e) The bifurcation diagram for $\Gamma_{2} \in[0.2,0.27]$

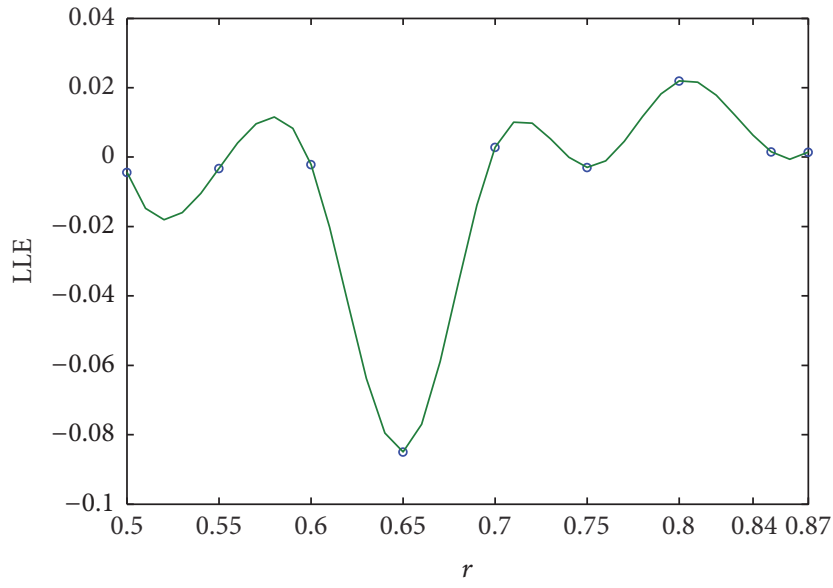

(b) The corresponding LLE for $c \in[0.5,0.87]$

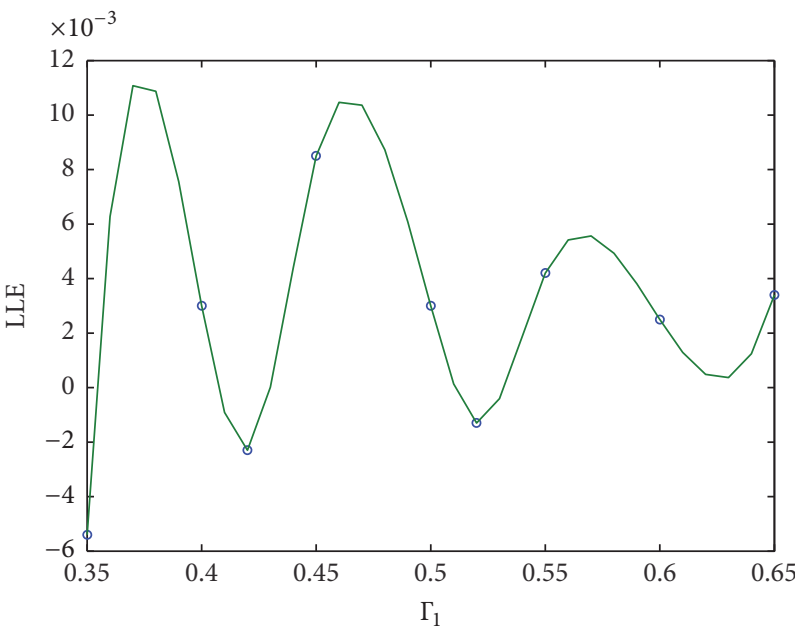

(d) The corresponding LLE for $\Gamma_{1} \in[0.35,0.65]$

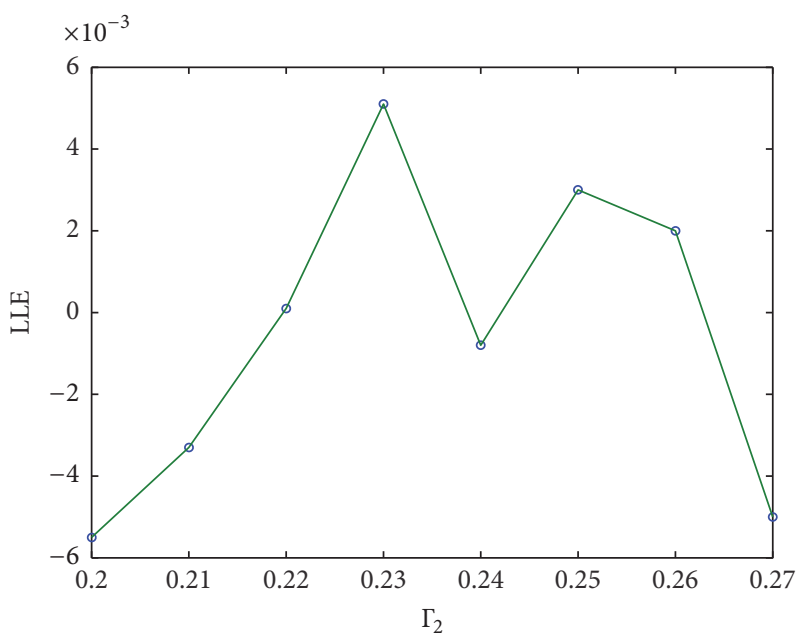

(f) The corresponding LLE for $\Gamma_{2} \in[0.2,0.27]$

FIGURE 8: The bifurcation and the corresponding LLE diagrams of system (2) with different parameters. 


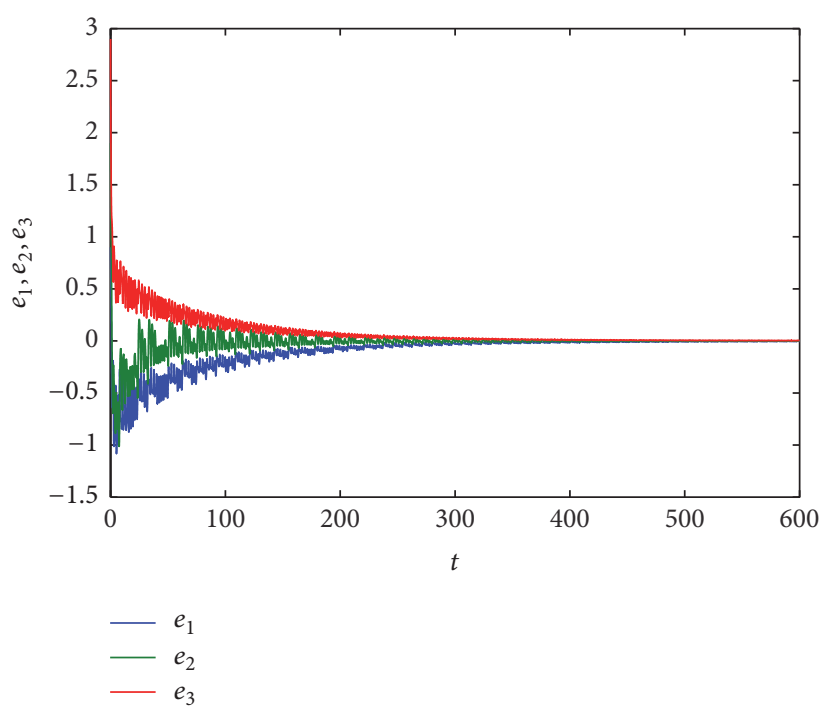

(a) The synchronization error curves

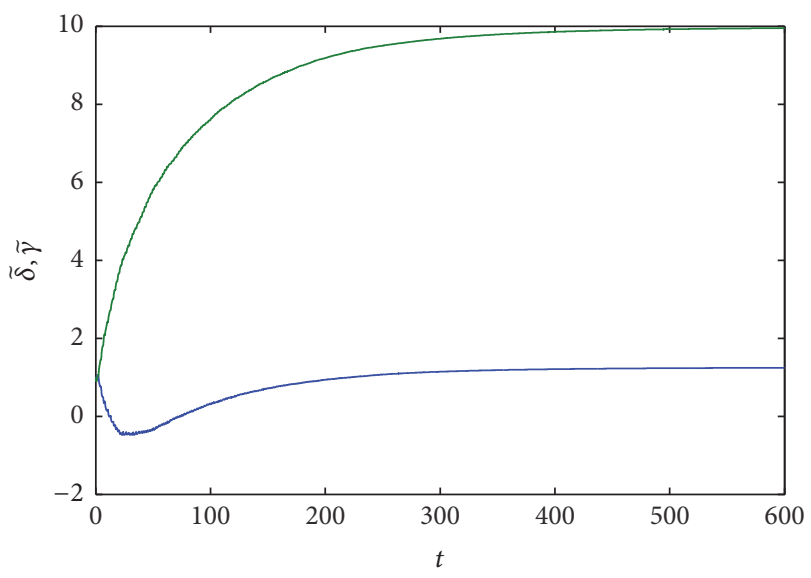

(b) The identification curves of the unknown parameters

FIgURE 9: The synchronization results of the numerical simulation.

Assume that $\lambda$ is one of the eigenvalues of matrix $\mathbf{A}$, and then the corresponding nonzero eigenvectors are $\zeta=\left(\zeta_{1}, \zeta_{2}\right.$, $\left.\zeta_{3}, \zeta_{4}, \zeta_{5}\right)^{\mathrm{T}}$; then we have

$$
\mathbf{A} \zeta=\lambda \zeta
$$

and the following relation can be easily obtained when conjugate transpose $\mathrm{H}$ is taken on both sides of (11)

$$
\overline{(A \zeta)^{\mathrm{T}}}=\bar{\lambda} \zeta^{\mathrm{H}}
$$

Using (11) multiplied left by $(1 / 2) \zeta^{\mathrm{H}}$ plus (12) multiplied right by $(1 / 2) \zeta$, we get

$$
\zeta^{\mathrm{H}}\left(\frac{1}{2} \mathbf{A}+\frac{1}{2} \mathbf{A}^{\mathrm{H}}\right) \zeta=\frac{1}{2}(\lambda+\bar{\lambda}) \zeta^{\mathrm{H}} \zeta .
$$

The above formula can be rewritten as the following form:

$$
\frac{1}{2}(\lambda+\bar{\lambda})=\frac{\zeta^{\mathrm{H}}\left((1 / 2) \mathbf{A}+(1 / 2) \mathbf{A}^{\mathrm{H}}\right) \zeta}{\zeta^{\mathrm{H}} \zeta} .
$$

By substituting the matrix A into (14), then we can have

$$
\frac{1}{2}(\lambda+\bar{\lambda})=\frac{1}{\zeta^{\mathrm{H}} \zeta} \zeta^{\mathrm{H}} \mathbf{B} \zeta
$$

where $\mathbf{B}=\operatorname{diag}(-1,-1,-1,0,0)$. Since $\lambda+\bar{\lambda} \leq 0$, then all the eigenvalues of matrix $\mathbf{A}$ satisfy the following relationship:

$$
|\arg (\lambda)| \geq \frac{\pi}{2}>\frac{\pi}{2} q, \quad(0<q<1) .
$$

According to the stability theory of fractional-order systems, then the error dynamical system (5) is asymptotically stable. Therefore

$$
\lim _{t \rightarrow \infty}\|e(t)\|=0,
$$

which means that the synchronization between the drive and response systems is realized. The proof is completed.
In numerical simulations, the real values of the uncertain parameters are $\delta=1.26, \gamma=10$ when $c=0.7764$, $\Gamma_{1}=0.5, \Gamma_{2}=0.25, q=0.98$. The initial conditions of the drive and response systems are $(0.1,0.1,0.1)$ and $(1,2,3)$, respectively. The synchronization results of the numerical simulation are depicted in Figure 9. From this we can see that the error variables tend to 0 , and the estimations of unknown parameters converge to their real values. These results demonstrate the effectiveness of the synchronization controllers and laws of unknown parameters.

\section{Conclusions}

In this paper, bifurcations and synchronization of a fractional-order Bloch system have been studied. Firstly, bifurcations of the system as every order is varied are obtained by the numerical simulations. Period-doubling and tangent bifurcations can be observed in the system. Meanwhile, bifurcations of the system with the variation of every system parameter are also determined by numerical computation. Besides the period-doubling and tangent bifurcations, limit cycles coexisting are also found for the fractional-order Bloch system. From these results, it can be seen clearly that the derivative orders are also the important parameters which affect the dynamics of the fractional-order Bloch system. The bifurcations of the system in such a parameter set are demonstrated in detail. Finally, based on the stability theory of fractional-order systems, the adaptive synchronization for the system with unknown parameters is realized by designing appropriate controllers. Numerical simulations are carried out to demonstrate the effectiveness and flexibility of the controllers.

\section{Conflicts of Interest}

The authors declare that they have no conflicts of interest. 


\section{Acknowledgments}

This work is supported by the National Natural Science Foundation of China (NSFC) under Grant nos. 11332008 and 11672218.

\section{References}

[1] M. M. Asheghan, S. S. Delshad, M. T. Hamidi Beheshti, and M. S. Tavazoei, "Non-fragile control and synchronization of a new fractional order chaotic system," Applied Mathematics and Computation, vol. 222, pp. 712-721, 2013.

[2] S. Dadras, H. R. Momeni, G. Qi, and Z.-l. Wang, "Fourwing hyperchaotic attractor generated from a new $4 \mathrm{D}$ system with one equilibrium and its fractional-order form," Nonlinear Dynamics, vol. 67, no. 2, pp. 1161-1173, 2012.

[3] C. Zeng, Q. Yang, and J. Wang, "Chaos and mixed synchronization of a new fractional-order system with one saddle and two stable node-foci," Nonlinear Dynamics, vol. 65, no. 4, pp. 457466, 2011.

[4] K. Diethelm and N. J. Ford, "Multi-order fractional differential equations and their numerical solution," Applied Mathematics and Computation, vol. 154, no. 3, pp. 621-640, 2004.

[5] K. Diethelm, N. J. Ford, A. D. Freed, and Y. Luchko, "Algorithms for the fractional calculus: a selection of numerical methods," Computer Methods in Applied Mechanics and Engineering, vol. 194, no. 6-8, pp. 743-773, 2005.

[6] T. T. Hartley, C. F. Lorenzo, and H. K. Qammer, "Chaos in a fractional order Chua's System," IEEE Transactions on Circuits and Systems I: Fundamental Theory and Applications, vol. 42, no. 8, pp. 485-490, 1995.

[7] Z.-M. Ge and C.-Y. Ou, "Chaos in a fractional order modified Duffing system," Chaos, Solitons and Fractals, vol. 34, no. 2, pp. 262-291, 2007.

[8] I. Grigorenko and E. Grigorenko, "Chaotic dynamics of the fractional Lorenz system," Physical Review Letters, vol. 91, no. 3, Article ID 034101, 2003.

[9] J. G. Lu, "Chaotic dynamics of the fractional-order Ikeda delay system and it synchronization," Physics Letters A, vol. 59, no. 2, pp. 301-305, 2006.

[10] W. Zhang, S. Zhou, H. Li, and H. Zhu, "Chaos in a fractionalorder Rössler system," Chaos, Solitons and Fractals, vol. 42, no. 3, pp. 1684-1691, 2009.

[11] V. Daftardar-Gejji and S. Bhalekar, "Chaos in fractional ordered Liu system," Computers and Mathematics with Applications, vol. 59, no. 3, pp. 1117-1127, 2010.

[12] S. Bhalekar, V. Daftardar-Gejji, D. Baleanu, and R. L. Magin, "Generalized fractional order bloch equation with extended delay," International Journal of Bifurcation and Chaos, vol. 22, no. 4, pp. 91-116, 2012.

[13] S. Bhalekar, V. Daftardar-Gejji, D. Baleanu, and R. Magin, "Fractional Bloch equation with delay," Computers and Mathematics with Applications, vol. 61, no. 5, pp. 1355-1365, 2011.

[14] S. Bhalekar, V. Daftardar-Gejji, D. Baleanu, and R. Magin, "Transient chaos in fractional Bloch equations," Computers and Mathematics with Applications, vol. 64, no. 10, pp. 3367-3376, 2012.

[15] R. L. Magin, O. Abdullah, D. Baleanu, and X. J. Zhou, "Anomalous diffusion expressed through fractional order differential operators in the Bloch-Torrey equation," Journal of Magnetic Resonance, vol. 190, no. 2, pp. 255-270, 2008.
[16] R. Magin, X. Feng, and D. Baleanu, "Solving the fractional order Bloch equation," Concepts in Magnetic Resonance Part A: Bridging Education and Research, vol. 34, no. 1, pp. 16-23, 2009.

[17] H. Nasr-eddine and H. Tarek, "Chaotic dynamics of the fractional order nonlinear Bloch system," Electronic Journal of Theoretical Physics, vol. 8, no. 25, pp. 233-244, 2011.

[18] I. Petráš, Fractional-Order Nonlinear Systems: Modeling, Anal$y$ sis and Simulation, Higher Education Press, Beijing, China, 2011.

[19] E. Hairer, S. P. Nørsett, and G. Wanner, Solving Ordinary Differential Equations I: Nonstiff Problems, vol. 8 of Springer Series in Computational Mathematics, Springer, Berlin, Germany, 2nd edition, 1993.

[20] E. Hairer and G. Wanner, Solving Ordinary Differential Equations II: Stiff and Differential- Algebraic Problems, vol. 14, Springer, Berlin, Germany, 1991.

[21] K. Diethelm, N. J. Ford, and A. D. Freed, "A predictor-corrector approach for the numerical solution of fractional differential equations," Nonlinear Dynamics, vol. 29, no. 1-4, pp. 3-22, 2002.

[22] A. Charef, H. H. Sun, Y.-Y. Tsao, and B. Onaral, "Fractal system as represented by singularity function," IEEE Transactions on Automatic Control, vol. 37, no. 9, pp. 1465-1470, 1992.

[23] M. S. Tavazoei and M. Haeri, "Limitations of frequency domain approximation for detecting chaos in fractional order systems," Nonlinear Analysis. Theory, Methods \& Applications, vol. 69, no. 4, pp. 1299-1320, 2008. 


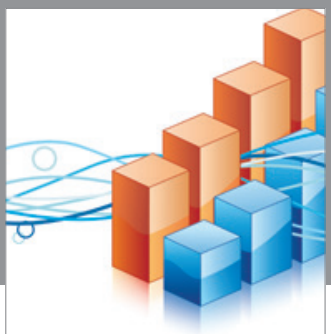

Advances in

Operations Research

vatem alat4

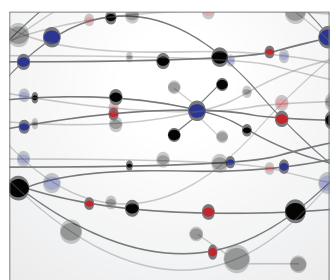

\section{The Scientific} World Journal
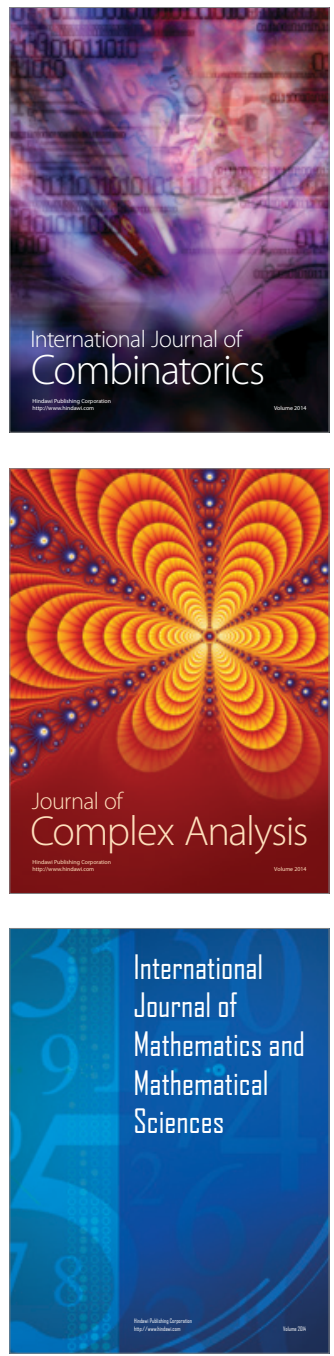
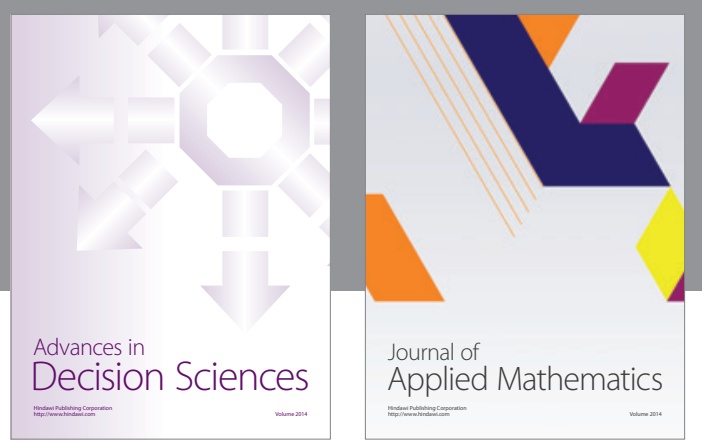

Algebra

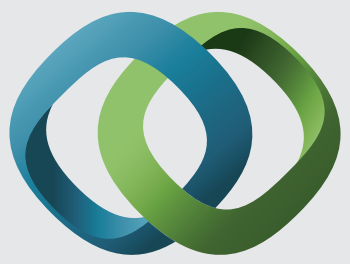

\section{Hindawi}

Submit your manuscripts at

https://www.hindawi.com
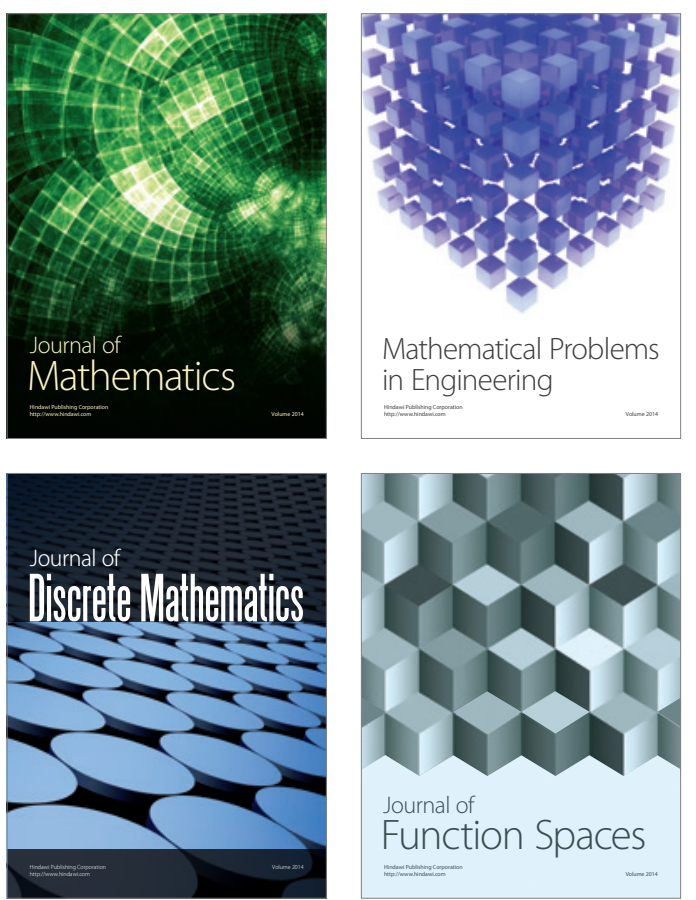

Mathematical Problems in Engineering
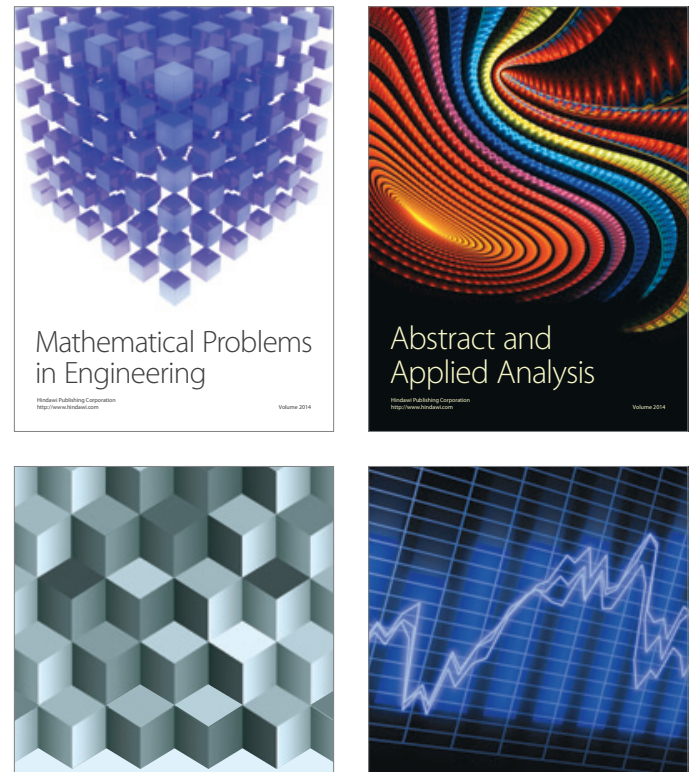

Journal of

Function Spaces

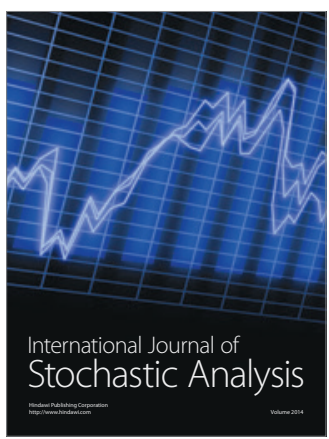

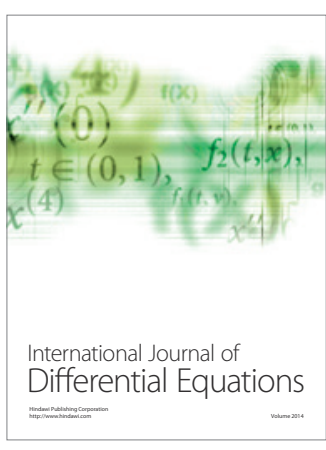
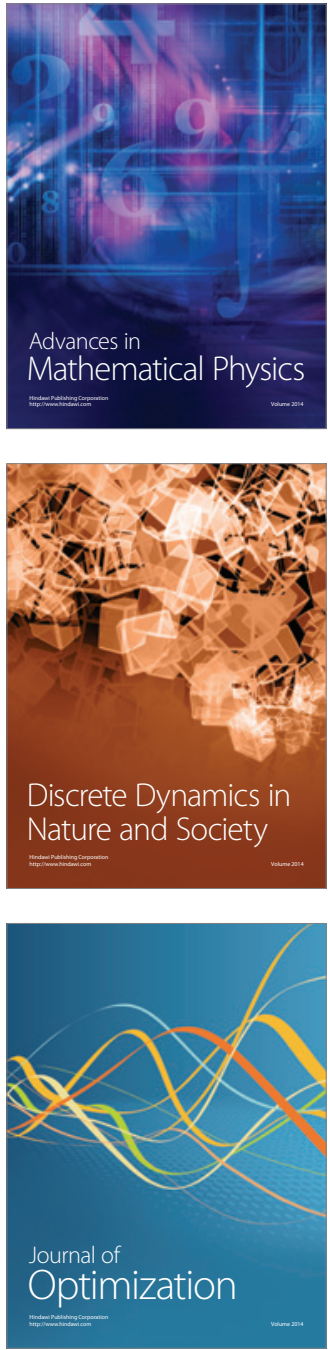